$A B$

LAL 94-59

August 1994

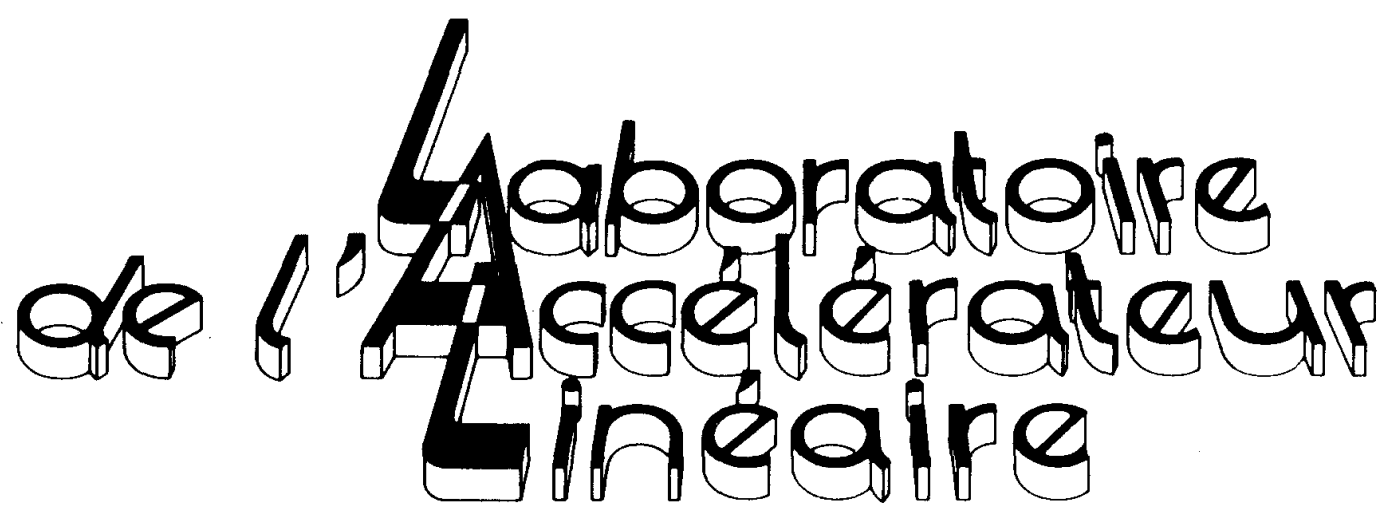

SEARCHES FOR NEW PARTICLES AT LEP

Swi34 44

Patrick JANOT

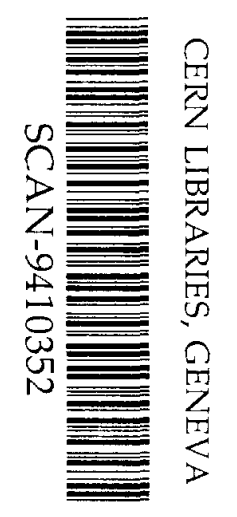

Invited talk given at the XVI International Conference on Neutrino Physics and Astrophysics Eilat, Read Sea, Israel, May 29 - June 3, 1994
U.E.R
de
l'Universite Paris - Sud

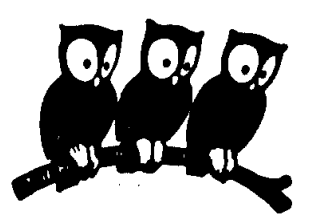
Institut National de Physique Nucleaire et
de Physique des Particules 



\title{
Searches for new particles at LEP
}

\author{
Patrick Janot \\ Laboratoire de l'Accélérateur Linéaire, IN2P3-CNRS \\ et Université de Paris-Sud, 91405 Orsay, France
}

30 August 1994

\begin{abstract}
The most recent developments in the domain of new particle searches at LEP are reviewed. In particular, the updates of the searches for the standard model Higgs boson and for the Higgs bosons of the MSSM are summarized. A new method is proposed to derive a 95\% C.L. limit on the standard model Higgs boson mass from the combinations of the analyses of the four LEP experiments. Finally, two monojet events from ALEPH with unexpectedly large transverse momenta and invariant masses are presented.
\end{abstract}





\title{
Searches for new particles at LEP
}

\author{
Patrick Janot \\ Laboratoire de l'Accélérateur Linéaire, IN2P3-CNRS \\ et Université de Paris-Sud, 91405 Orsay, France
}

The most recent developments in the domain of new particle searches at LEP are reviewed. In particular, the updates of the searches for the standard model Higgs boson and for the Higgs bosons of the MSSM are summarized. A new method is proposed to derive a 95\% C.L. limit on the standard model Higgs boson mass from the combinations of the analyses of the four LEP experiments. Finally, two monojet events from ALEPH with unexpectedly large transverse momenta and invariant masses are presented.

\section{INTRODUCTION}

After a few months of data taking at LEP, most of the searches for pair-produced particles reached their kinematical limit. Since then, the interest in this type of research considerably decreased, leading now to a very low level of activity in this domain. Although a few updates and even a few new searches for very peculiar final states (heavy scalar search from DELPHI, longlived heavy neutrino search from DELPHI, scalar top quark search from OPAL) have been reported to me with still negative results, no details about these analyses will be presented here.

However, an active search is still pursued by each of the four LEP collaborations in at least three domains:

- the search for the standard model Higgs boson, essentially not constrained by the LEP precision measurements [1] and far from its kinematical limit;

- the search for the Higgs bosons of the minimal supersymmetric extension of the standard model (the MSSM), motivated by the prediction of at least one light scalar Higgs boson (below $95-100 \mathrm{GeV} / \mathrm{c}^{2}[2]$ ) and also not yet kinematically limited;

- the stimulating search for the unexpected.
Each of these three points are reviewed in the following sections. A combined lower limit on the standard model Higgs boson mass is extracted from the four LEP experiment results in Section 2, taking a particular care in preserving the $95 \%$ confidence level of this limit. This limit is reinterpreted for the Higgs bosons of the MSSM in Section 3. Finally, two monojet events with unexpected characteristics are presented and tentatively interpreted in Section 4.

\section{THE STANDARD MODEL HIGGS BOSON}

2.1. Production, decays and final states

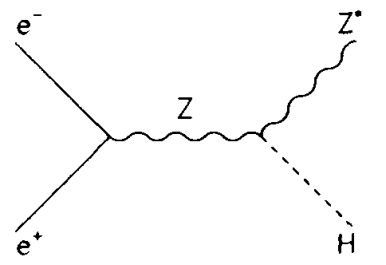

Figure 1. Feynman diagram for the Bremsstrahlung process $\mathrm{e}^{+} \mathrm{e}^{-} \rightarrow \mathrm{HZ}^{*}$;

At the $\mathrm{Z}$ peak, the standard model Higgs boson is expected to be produced mainly by the socalled Bremsstrahlung process $\mathrm{e}^{+} \mathrm{e}^{-} \rightarrow \mathbf{H Z}^{*}$, the Feynman diagram of which is shown in Fig. 1. 
Another production mechanism, $\mathrm{e}^{+} \mathrm{e}^{-} \rightarrow \mathrm{H} \gamma$ via an intermediate top-quark loop, is expected to occur with a much lower rate (see Fig. 2) and with a prominent background from $\mathrm{e}^{+} \mathrm{e}^{-} \rightarrow \mathrm{q} \bar{q} \gamma$. It is therefore not considered in the following.

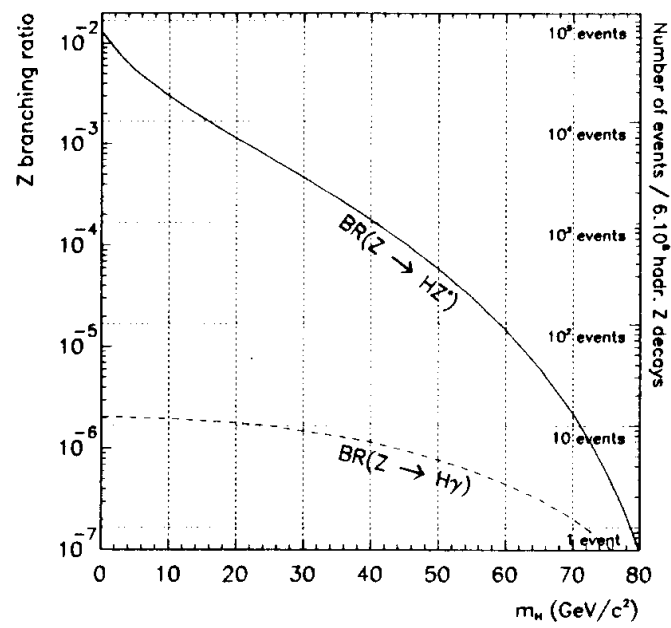

Figure 2. Branching ratio of the $\mathrm{Z}$ decay into $\mathrm{HZ}^{*}$ (full line, left axis) and number of $\mathrm{e}^{+} \mathrm{e}^{-} \rightarrow \mathrm{HZ}^{*}$ events expected in the LEP sample corresponding to six million hadronic $\mathrm{Z}$ decays (right axis), as a function of the Higgs boson mass. Also indicated is the branching ratio of the $\mathrm{Z}$ decay into $\mathrm{H} \gamma$ via a top-quark loop (dashed line).

At the time of the conference, the data sample analyzed by the four LEP collaborations amounted to almost six million hadronic $\mathrm{Z}$ decays:

- 950,000 events from DELPHI; corresponding to their 1991-92 data [3];

- 1,050,000 events from L3, corresponding to their 1990-92 data[4];

- 1,900,000 events from OPAL, corresponding to their 1990-1993 data [5];

- 1,950,000 events from ALEPH, corresponding to their 1989-1993 data $[6,7]$.
As can be seen in Fig. 2, almost 40 events are expected to be produced in this sample for $m_{\mathrm{H}}=$ $65 \mathrm{GeV} / c^{2}$ and 12 events for $m_{\mathrm{H}}=70 \mathrm{GeV} / c^{2}$. Unfortunately, not all the final states can be disentangled from the background: since the standard model Higgs boson decays in $\sim 9 \%$ of the cases into $\tau^{+} \tau^{-}[8]$ and in the remaining $\sim 91 \%$ into hadrons (of which $95 \%$ into $b \bar{b}$ ), most of the final states are either purely hadronic when $\mathrm{Z}^{*} \rightarrow \mathrm{q} \overline{\mathrm{q}}$, or made of a tau pair accompanied by a hadronic system, both of them being overwhelmed with the huge $\mathrm{e}^{+} \mathrm{e}^{-} \rightarrow$ hadrons background.

Actually only $24 \%$ of the final states, leading to well identifiable topologies, have been used in the search for the Higgs boson by the four experiments:

- the $(H \rightarrow$ hadrons $)\left(Z^{*} \rightarrow \nu \bar{\nu}\right)$ final state, turning into two acoplanar jets accompanied with missing energy, hereafter called the $\mathbf{H} \nu \bar{\nu}$ channel;

- the $(\mathrm{H} \rightarrow$ hadrons $)\left(\mathrm{Z}^{*} \rightarrow \mathrm{I}^{+} \mathrm{I}^{-}\right)$final state, with $\mathrm{l}=\mathrm{e}$ or $\mu$, producing two energetic leptons isolated from the accompanying hadronic system, called the $\mathrm{HI}^{+} \mathrm{l}^{-}$channel.

This leaves only 10 events (respectively 3 events) available for the search when $m_{\mathrm{H}}=65 \mathrm{GeV} / \mathrm{c}^{2}$ (resp. $70 \mathrm{GeV} / c^{2}$ ), thus almost ruling out the possibility of discovering such a heavy Higgs boson with the statistics presently accumulated at LEP.

\subsection{The $\mathbf{H} \nu \bar{\nu}$ channel}

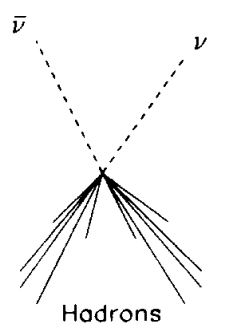

The topology of interest here is an acoplanar pair of jets accompanied by missing energy. Since the total visible mass is expected to be the Higgs boson mass, a good resolution both for the energies and for the directions of 
the jets is of prime importance to increase the signal-to-background ratio. The resulting invariant mass resolutions for masses around $60 \mathrm{GeV} / c^{2}$ are shown in Table 1 for the four LEP detectors.

Table 1

Invariant mass resolutions, in $\mathrm{GeV} / c^{2}$, for masses around $60 \mathrm{GeV} / c^{2}$.

\begin{tabular}{rrrrr}
\hline Detector & DELPHI & $\overline{\text { L3 }}$ & OPAL & ALEPH \\
\hline$\sigma_{M}$ & 10 & 8 & 7 & 5 \\
\hline
\end{tabular}

Since the events liable to produce missing energy, but arising from different sources, are common to all experiments, it is not surprising that similar variables be used to discriminate signal and backgrounds. First, hadronic final states with missing energy are selected by requiring a large number of particles to be detected and, in general, a total visible mass smaller than $\sim 70 \mathrm{GeV} / c^{2}$ to be measured. The various potential background sources are taken care of in the following way:

1. the dead zones of the detectors are avoided, especially near the vacuum chamber, using the energy measured in the forward subdetectors and/or the direction of the trust axis of the events;

2. $\gamma \gamma$ collisions are recognized with their small visible mass and transverse momentum (typically smaller than $25 \mathrm{GeV} / c^{2}$ and $5 \mathrm{GeV} / c$, respectively);

3. $\mathrm{e}^{+} \mathrm{e}^{-} \rightarrow \mathrm{b} \overline{\mathrm{b}}$ events with semi-leptonic decays, therefore with missing energy coming from the decay neutrinos, have two collinear jets in the final state (with a typical acollinearity angle above 150 to $170^{\circ}$ );

4. hadronic events accompanied by an undetected energetic photon from initial state radiation (ISR) are expected to present a missing momentum direction along the beam axis (below 20 to $25^{\circ}$ );
5. $\mathrm{e}^{+} \mathrm{e}^{-} \rightarrow \mathrm{b} \overrightarrow{\mathrm{b}}$ events with ISR and with semileptonic decays are still characterized by two coplanar jets in the final states (with a typical acoplanarity angle above 160 to $\left.175^{\circ}\right)$

6. $\mathrm{e}^{+} \mathrm{e}^{-} \rightarrow \mathrm{bb} g$ events, leading to three jets in the final state, are characterized either by their thrust value, or by their nonisolated missing momentum (in cones of half-opening angle $20,30,60$ or $90^{\circ}$ ), or by their planarity (the typical sum of the three jet-jet angles is above 340 to $350^{\circ}$ );

7. Finally, $\mathrm{e}^{+} \mathrm{e}^{-} \rightarrow \mathrm{b} \bar{b} g(\gamma)$ events (with ISR) are compatible with a four-body final state.

Amazingly enough, in spite of this common underlying physics, the analysis techniques vary a lot from an experiment to the other.

The most involved technique has been developed by the DELPHI collaboration, with two different analyses. The first one is based on a neural network with fifteen input variables, two intermediate layers and one output variable. In the second approach, called probabilistic approach, a probability is determined for each event from the fraction of Monte Carlo background events for which fourteen variables are found to be "more exotic" than for the current event. In both cases, after a preselection with cuts on about ten variables, such as the visible mass, the acollinearity angle or the b-quark content of the events, and a final cut either on the output variable or on the probability, the location of which is determined on Monte Carlo to lead to less than one background event expected, no events are found in the data with an efficiency for a $60 \mathrm{GeV} / c^{2}$ Higgs boson between 25 and $30 \%$. This efficiency increases to $35 \%$ when the two analyses are combined, corresponding to 0.94 signal events expected, and $1.1_{-0.3}^{+0.6}$ background events are expected to be found in the data.

In contrast, the L3 analysis is particularly straightforward: only seven variables are used (visible mass, transverse momentum, direction of the missing momentum, acoplanarity and 

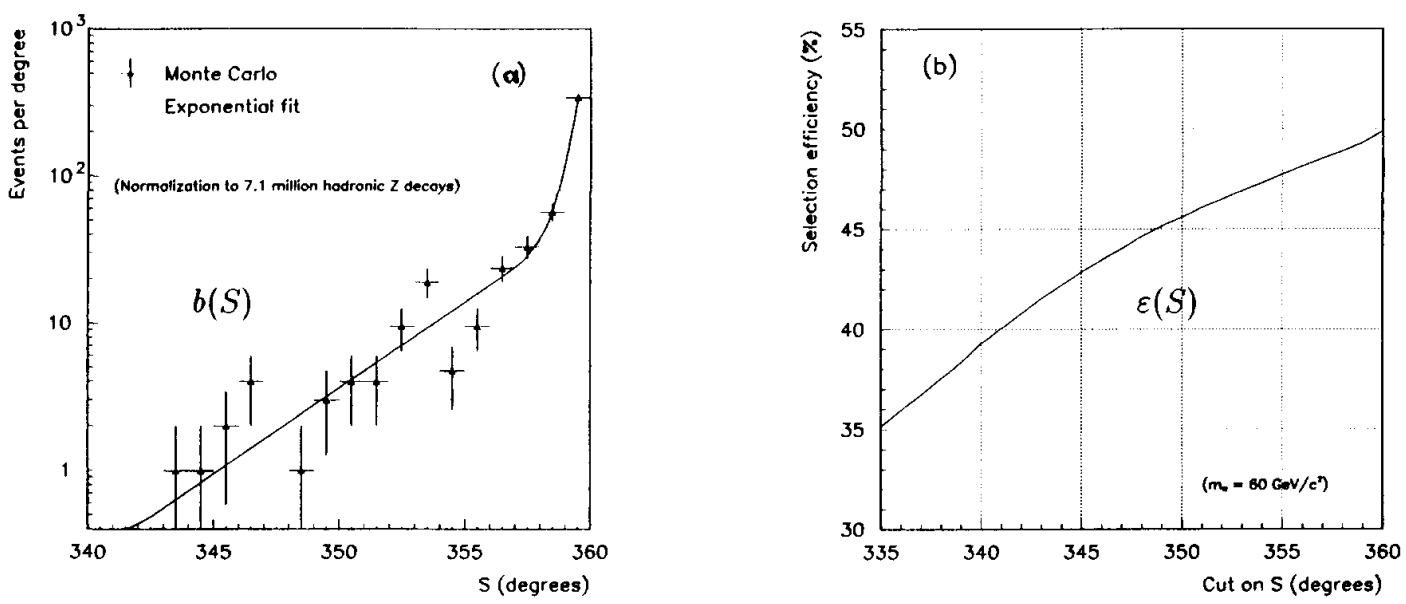

Figure 3. Variables used in the ALEPH cut location optimization, for the sum $S$ of the three jet-jet angles: (a) distribution and fit of the expected background $b(S)$; and (b) selection efficiency $\varepsilon(S)$;

acollinearity angle, and isolation of the missing momentum in two different cones) in a set of successive cuts, leading to no events in the data. An efficiency of $44.5 \%$ for a $60 \mathrm{GeV} / c^{2}$ Higgs boson is achieved, corresponding to 1.32 signal events expected. However, the background study is based on a Monte Carlo sample of only 1.1 million hadronic $\mathrm{Z}$ decays, while other backgrounds have not been yet considered [9]. An accurate background estimate is therefore difficult to obtain: less than $2.8 \mathrm{Z} \rightarrow \mathrm{q} \overline{\mathrm{q}}$ events are expected at the $95 \%$ confidence level.

OPAL have chosen a much more conservative approach: the background study is based on a Monte Carlo sample equivalent to over 35 million hadronic $\mathrm{Z}$ decays, and severe topological cuts are applied in order to select the fewest possible events from this sample. The consequence is of course a lower selection efficiency of $32.4 \%$ for a $60 \mathrm{GeV} / c^{2}$ Higgs boson, corresponding to 1.75 signal events expected, but this technique allowed the analysis not to be modified with respect to the preceeding year: only $0.22_{-0.05}^{+0.08} \mathrm{Z} \rightarrow \mathrm{q} \overline{\mathrm{q}}$ are expected to be found in the present data sample.

An even more sophisticated method [10] has been used by ALEPH to determine the cut locations in an unbiased way (i.e. not influenced by the data). Monte Carlo samples corresponding to typically five times the total integrated luminosity have been produced for all known background processes. Large signal Monte Carlo samples, for several Higgs boson mass values, have also been produced. The cuts have been determined by minimizing the average value $\bar{N}_{95}(x)$ of the $95 \%$ C.L. upper limit on the number of signal events produced which would be obtained with an infinity of gedanken experiments in the absence of any signal contribution:

$$
\begin{aligned}
\overline{\mathrm{N}}_{95}(x)=\frac{e^{-b(x)}}{\varepsilon(x)}\{3.00 & +4.74 b(x) \\
+ & 6.30 \frac{b^{2}(x)}{2 !}+7.75 \frac{b^{3}(x)}{3 !} \\
& +\ldots\} .
\end{aligned}
$$

In this formula, $x$ is the location of the cut and $b(x)$ is the number of background events expected when the cut is applied. This number is determined from the background Monte Carlo distribution of the variable $x$ obtained when all other cuts have been applied, smoothed and extrapolated so that an analytical representation of $b(x)$ is available (see Fig. 3a). The numbers 3.00, 4.74, $6.30, \ldots$ are the $95 \%$ C.L. upper limits on the number of signal events expected if $0,1,2, \ldots$ background events are observed, while the coef- 
Table 2

Numbers of hadronic $\mathrm{Z}$ decays analyzed, numbers of background events expected, selection efficiencies for a $60 \mathrm{GeV} / c^{2}$ Higgs boson and corresponding numbers of signal events expected, in the $\mathrm{H} \nu \bar{\nu}$ channel.

\begin{tabular}{rrrrr}
\hline Expt. & $\# \mathrm{Z} \rightarrow \mathrm{q} \overline{\mathrm{q}}$ & Background & $\varepsilon_{60}(\%)$ & $N_{60}$ \\
\hline DELPHI & $966 \mathrm{~K}$ & $1.1_{-0.3}^{+0.6}$ & 35.0 & 0.94 \\
L3 & $1062 \mathrm{~K}$ & $\leq 2.8$ & 44.5 & 1.32 \\
OPAL & $1900 \mathrm{~K}$ & $0.22_{-0.05}^{+0.08}$ & 32.4 & 1.75 \\
ALEPH & $1936 \mathrm{~K}$ & $0.26_{-0.10}^{+0.15}$ & 42.0 & 2.32 \\
\hline Total & $5864 \mathrm{~K}$ & $1.58_{-0.32}^{+1.25}$ & 38.2 & $\mathbf{6 . 3 3}$ \\
\hline
\end{tabular}

ficients of these numbers are the corresponding Poisson probabilities. Finally, $\varepsilon(x)$ is the acceptance of the search for the signal, as a function of the cut location $x$ (see Fig. 3b). The minimization of $\overrightarrow{\mathrm{N}}_{95}(x)$ (see Fig. 4) is done after the absolute normalization of $b(x)$ is reduced according to the visible mass resolution.

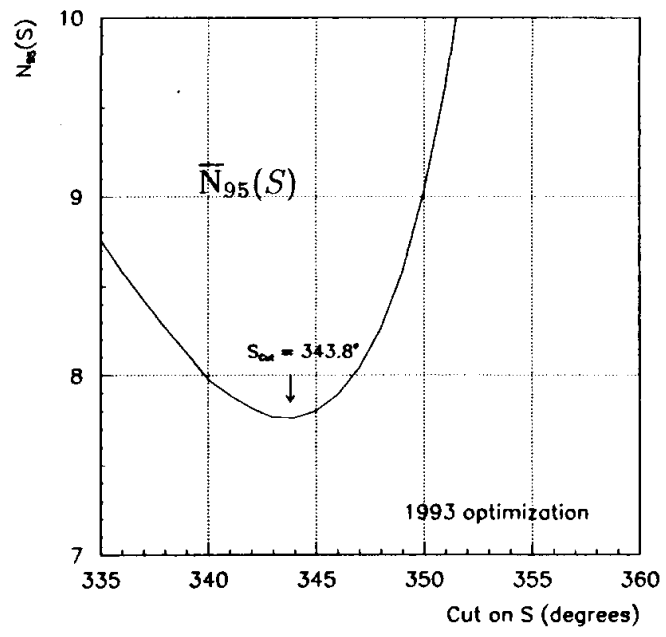

Figure 4. Example of cut optimization, applied to the variable $S$. The minimum of $\bar{N}_{95}(S)$ at $343.8^{\circ}$ is obtained after the mass resolution has been accounted for.

After the procedure has been applied to several variables, the resulting number of background events is $0.26_{-0.10}^{+0.15}$ around $60 \mathrm{GeV} / \mathrm{c}^{2}$. No events are found in the data and the efficiency achieved for a $60 \mathrm{GeV} / \mathrm{c}^{2}$ Higgs boson amounts to $42 \%$, corresponding to 2.32 signal events expected.
A summary of the results of the four analyses is reported in Table 2 . It should be mentioned, however, that a search for the $\left(\mathrm{H} \rightarrow \tau^{+} \tau^{-}\right)\left(\mathrm{Z}^{*} \rightarrow \nu \bar{\nu}\right)$ final state has also been used by L3, increasing by less than $10 \%$ the number of signal events expected. A comment about this is made in Section 2.4 .

\subsection{The $\mathrm{HI}^{+} \mathrm{I}^{-}$channel}

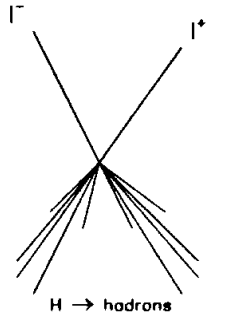

The presence of two energetic, isolated, and thus well identifiable leptons renders remarkably simple and efficient the selection of this final state. But every rose has its thorn: an important background is expected from the four-fermion electroweak production, the dominant Feynman diagrams of which are shown in Fig. 5a. For instance, ALEPH observe 29 such events, in agreement with the FERMISV [11] prediction of 28.3 events, with a hadronic mass distribution shown in Fig. 5b.

Table 3

Recoil mass resolutions (in $\mathrm{GeV} / c^{2}$ ) for $m_{\mathrm{H}}=$ $60 \mathrm{GeV} / c^{2}$ in the $\mathrm{H} \mu^{+} \mu^{-}$channel.

\begin{tabular}{rrrrr}
\hline Detector & DELPHI & L3 & OPAL & ALEPH \\
\hline$\sigma_{M}$ & 1.2 & 0.7 & 1.0 & 0.4 \\
\hline
\end{tabular}

Fortunately enough, this is compensated by the excellent Higgs boson mass resolution achieved when computed as recoiling against the lepton pair (see Table 3):

$m_{\mathrm{H}^{2}}^{2}=s+m_{1^{+1^{-}}}^{2}-2 \sqrt{s}\left(E_{\mathrm{l}^{+}}+E_{\mathrm{l}^{-}}\right)$, 


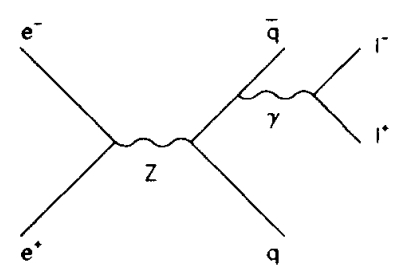

(a)

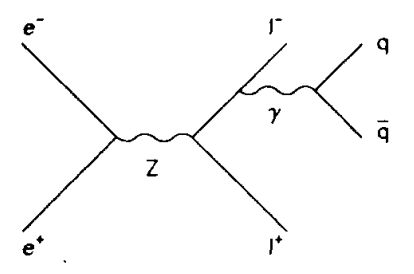

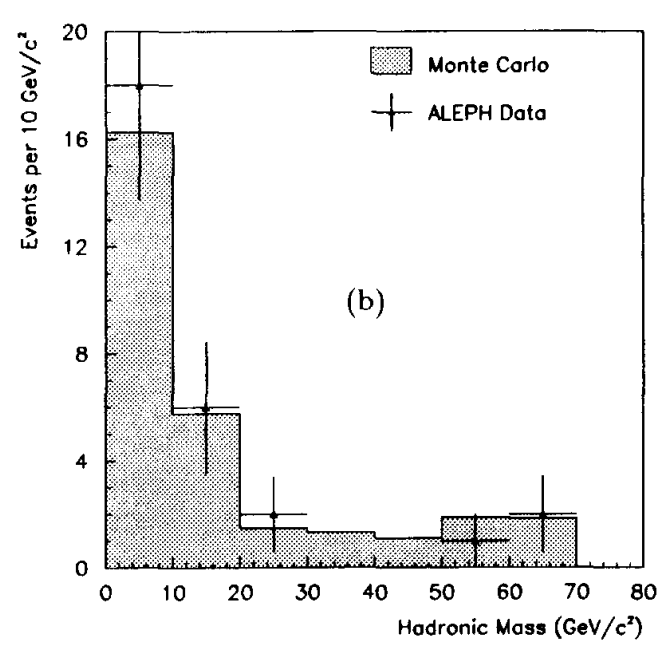

Figure 5. (a) Dominant Feynman diagrams for the four-fermion process $\mathrm{e}^{+} \mathrm{e}^{-} \rightarrow \mathrm{l}^{+} \mathrm{l}^{-} \mathrm{q} \overline{\mathrm{q}}$; (b) Distribution of the hadronic mass for the ALEPH $\mathrm{l}^{+} \mathrm{I}^{-} \mathrm{q}$ q events (triangles with error bars) and for the Monte Carlo prediction (shaded area) with an absolute normalization.

where $m_{1+1^{-}}^{2}$ is the invariant mass squared of the lepton pair, $E_{1+}$ and $E_{1-}$ are the lepton energies, and $\sqrt{s}$ is the centre-of-mass energy.

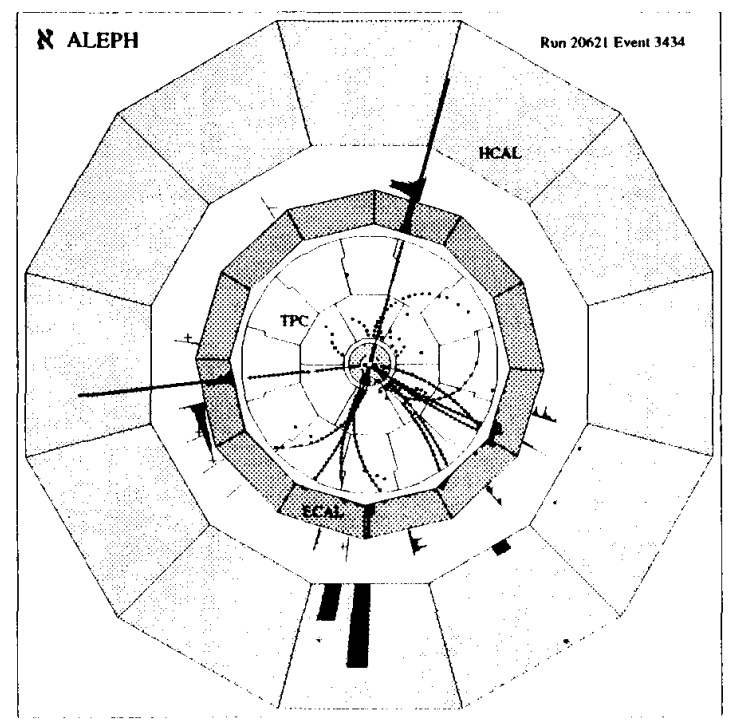

Figure 6. A $r \varphi$ view of the ALEPH detector for a $\mathrm{e}^{+} \mathrm{e}^{-} \rightarrow \mathrm{e}^{+} \mathrm{e}^{-} \mathrm{q} \overline{\mathrm{q}}$ event.
An example of $\mathrm{a} \mathrm{e}^{+} \mathrm{e}^{-} \mathrm{q} \overline{\mathrm{q}}$ event is shown in Fig. 6, and a summary of the results of the four analyses is reported in Table 4 , after a cut at $40 \mathrm{GeV} / c^{2}$ on the mass recoiling against the two leptons, and a loose b-tagging requirement (yet in the ALEPH analysis only) aimed at increasing the signal-tonoise ratio.

The two L3 candidates, the OPAL candidate and the ALEPH candidate correspond to Higgs boson masses of $70.4 \pm 0.7,67.6 \pm 0.7,61.2 \pm 1.0$ and $51.4 \pm 0.4 \mathrm{GeV} / c^{2}$, respectively.

\subsection{Towards a combined $95 \%$ C.L. mass limit?}

Usually, a combined limit on the standard model Higgs boson mass is defined as the mass for which the sum of the numbers of signal events expected by each of the four experiments amounts to 3.0 , at least when no events are observed in the data or when they are "far enough" from the limit [12]. This simple definition is used de facto by each individual experiment when combining the $\mathrm{H} \nu \bar{\nu}$ and the $\mathrm{HI}^{+} \mathrm{I}^{-}$results. 
Table 4

Selection efficiencies for a $60 \mathrm{GeV} / \mathrm{c}^{2}$ Higgs boson and corresponding numbers of signal events expected, numbers of background events expected and observed, in the $\mathrm{Hl}^{+} \mathrm{l}^{-}$channel.

\begin{tabular}{rrrrr}
\hline Expt. & $\varepsilon_{60}(\%)$ & $N_{60}$ & Expected & Observed \\
\hline DELPHI & 43.0 & 0.39 & $3.6 \pm 1.6$ & 0 \\
L3 & 48.8 & 0.49 & $2.5 \pm 0.4$ & 2 \\
OPAL & 38.0 & 0.68 & $0.8 \pm 0.2$ & 1 \\
ALEPH & 45.0 & 0.75 & $1.1 \pm 0.2$ & 1 \\
\hline Total & 43.1 & $\mathbf{2 . 2 9}$ & $8.0 \pm 1.0$ & $\mathbf{4}$ \\
\hline
\end{tabular}

It leads to the following published limits (see Table 5), where all selected events are assumed to arise from the signal. In L3 and ALEPH, the mass resolution and the mass of the candidates is exploited in the limit determination as explained in Ref. [13]. OPAL ignore the mass information of the $\mathrm{HI}^{+} \mathrm{I}^{-}$candidate, and DELPHI have no candidate at high mass. The $\left(\mathrm{H} \rightarrow \tau^{+} \tau^{-}\right)\left(Z^{*} \rightarrow\right.$ $\nu \tilde{\nu})$ analysis is included in the L3 limit.

Table 5

Published 95\% C.L. limits on the standard model Higgs boson mass, in $\mathrm{GeV} / \mathrm{c}^{2}$.

\begin{tabular}{rrrrr}
\hline Expt. & DELPHI & L3 & OPAL & ALEPH \\
\hline Limit & 55.7 & 57.7 & 56.9 & 60.3
\end{tabular}

However, if this method guarantees the achievement of a mass limit, it does not automatically provide a confidence level of $95 \%$. For instance, with this method, an analysis leading to a huge number of background events expected would degrade, on average, the $95 \%$ C.L. mass limit obtained without including this analysis. This is independent of the number of events observed in the actual experiments, which must not be accounted for in an a priori choice of analyses to combine. The following philosophy has therefore been followed:

1. to avoid any kind of biases, only the Monte Carlo expectations are used in this a priori decision of which analysis is kept to derive the limit;
2. the average value $\overline{\mathrm{N}}_{95}$ of the $95 \%$ C.L. limit on the signal cross-section, as defined in Section 2.2 , is computed from the number of background events expected in each analysis, conservatively increased by one standard deviation as indicated in Tables 2 and 4 , taking into account the mass resolution and assuming a flat mass distribution of this background. This is done with a Higgs boson mass of $60 \mathrm{GeV} / c^{2}$ for each individual experiments, and of $65 \mathrm{GeV} / c^{2}$ for the overall combination;

3. any analysis that would degrade, on average, the limit - or, equivalently, that makes $\overline{\mathbf{N}}_{95}$ increase - is not used in the combination.

As can be seen in Fig. 7, the average 95\% C.L. limit on the Higgs boson mass always improves when the $\mathrm{Hl}^{+} \mathrm{I}^{-}$channel is combined with the $\mathrm{H} \nu \bar{\nu}$ channel, provided that the mass information of the candidate events be used. The OPAL limit is expected to degrade (and it actually does) when combining the $\mathrm{Hl}^{+} \mathrm{l}^{-}$channel without this mass information, as done in Ref. [5]. The L3 limit is also expected to be worsened (on average) when the $\left(\mathrm{H} \rightarrow \tau^{+} \tau^{-}\right)\left(Z^{*} \rightarrow \nu \bar{\nu}\right)$ is added.

When the mass information is exploited in OPAL analysis, when the $\tau^{+} \tau^{-} \nu \bar{\nu}$ channel of $\mathrm{L} 3$ is ignored, and when the correct branching ratio of the Higgs boson into $\tau^{+} \tau^{-}$and the recent estimate of the top-quark mass [14] are used to compute the numbers of signal events expected, each individual $95 \%$ C.L. mass limit can be estimated as reported in Table 6 . 


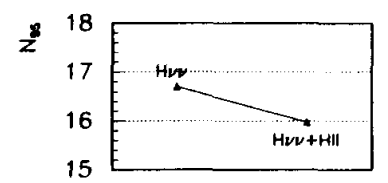

DELPHI

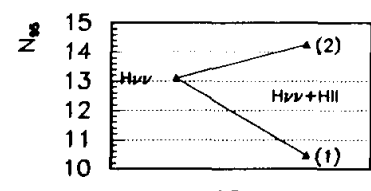

OPAL

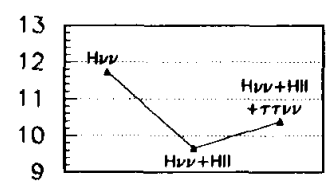

L3

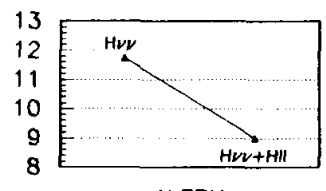

ALEPH
Figure 7. Evolution of $\bar{N}_{95}$ when combining the $\mathrm{HI}^{+} \mathrm{l}^{-}$channel with the $\mathrm{H} \nu \bar{\nu}$ channel, in each of the four experiments. The effect of the $(\mathrm{H} \rightarrow$ $\left.\tau^{+} \tau^{-}\right)\left(Z^{*} \rightarrow \nu \bar{\nu}\right)$ channel is also shown for $\mathbf{L 3}$. For OPAL, the result is plotted when the mass information of the candidate events is (1) or is not (2) used.

Table 6

My personal estimate of the $95 \%$ C.L. limits on the standard model Higgs boson mass, in $\mathrm{GeV} / \mathrm{c}^{2}$.

\begin{tabular}{rrrrr}
\hline Expt. & DELPHI & L3 & OPAL & ALEPH \\
\hline Limit & 55.6 & 57.0 & 58.5 & 60.2 \\
\hline
\end{tabular}

The same procedure can now be tried towards a combination of the four experiment results. Since the individual analyses have not been optimized in view of such a combination, it is not surprising that some of them have to be ignored in the derivation of the final $95 \%$ C.L. limit, preferably those with the smallest relative efficiency, the largest expected background or the worst mass resolution. It is shown in Fig. $8 \mathrm{a}$ that three of them have actually to be rejected in order not to degrade the average value of the limit.

This uncomfortable situation could be improved by optimizing the individual analyses as if six million hadronic $Z$ decays were available in each experiment, so that they become sensitive to a $65 \mathrm{GeV} / c^{2}$ Higgs boson. When playing this game with the ALEPH optimization method des- cribed in Section 2.2, the conclusion is that the efficiencies have to be lowered by approximately $15 \%$ while the background levels are divided by $\sim 3$. The resulting combination curve, assuming that the same occurs in the other experiments, is shown in Fig. 8b: only one analysis have to be rejected in that case, and the combined limit improves on average.
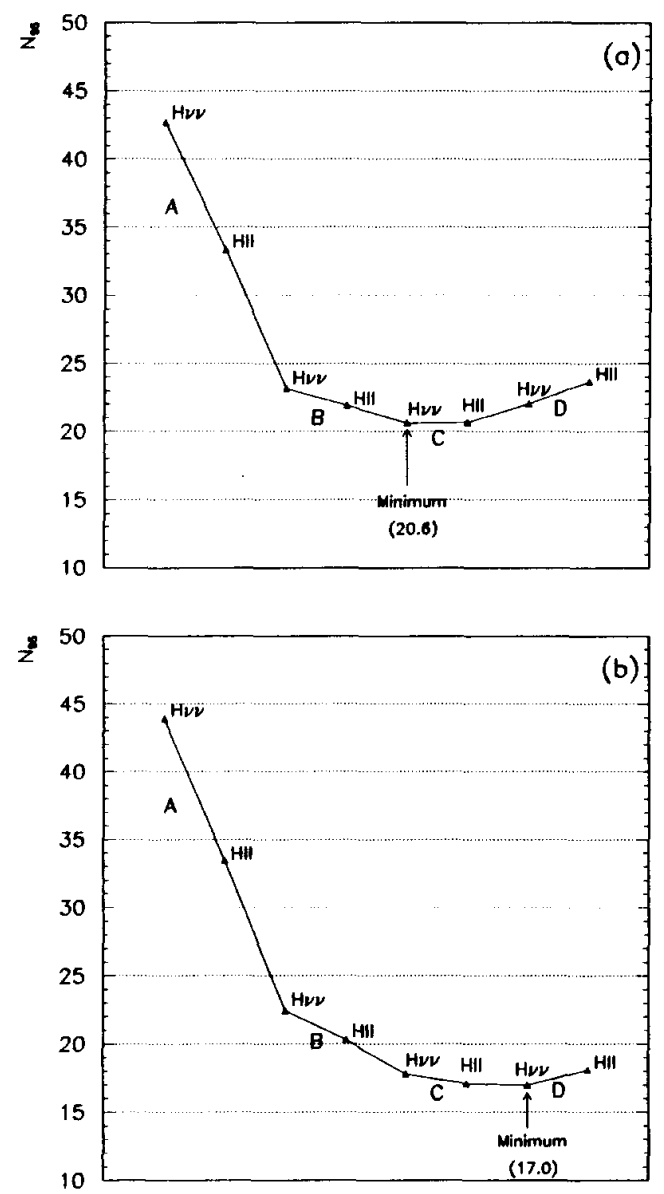

Figure 8. Evolution of $\bar{N}_{95}$ when combining the four experiments denoted here A, B, C, D. The chosen combination order is the order leading to the best $95 \%$ C.L. limit, (a) with the original analyses and (b) with the analyses optimized as indicated in the text. 
The actual combined lower limit on the standard model Higgs boson mass thus obtained from the four LEP experiments is $63.9 \mathrm{GeV} / c^{2}$ at the 95\% confidence level, as shown in Fig. 9. It should be mentioned, however, that this limit is very close to the OPAL $\mathrm{Hl}^{+} \mathrm{l}^{-}$candidate: it would suddenly become $61 \mathrm{GeV} / c^{2}$ if the confidence level required were $97 \%$ instead of $95 \%$.

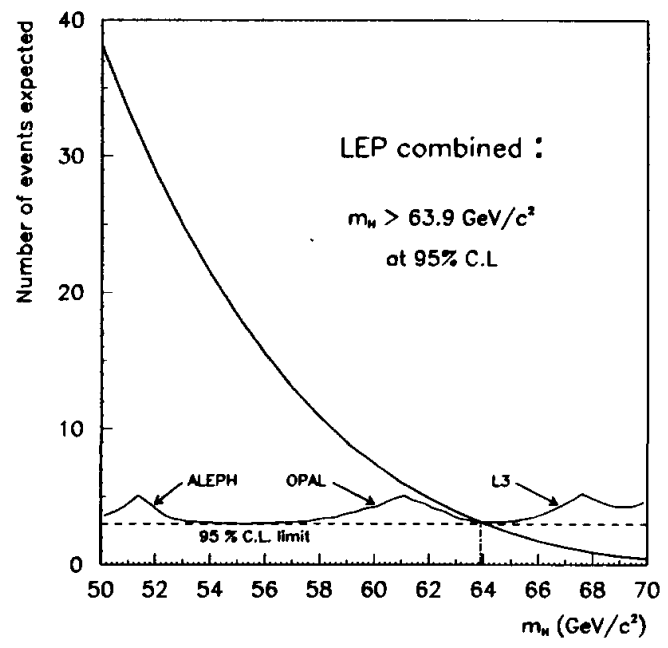

Figure 9. Total number of events expected from the seven kept analyses, and after optimization [see text], and $95 \%$ C.L. combined lower limit on the standard model Higgs boson mass. Also shown is the effect of the $\mathrm{HI}^{+} \mathrm{I}^{-}$candidates of ALEPH, OPAL and L3.

When the data collected by DELPHI [15] and $\mathbf{L 3}$ [9] in 1993 - made available after this conference and still preliminary - are included, no new candidates appear with a mass below $70 \mathrm{GeV} / c^{2}$ in any channel, and the combined 95\% C.L. limit on the Higgs boson mass, derived using the method presented above, becomes $64.3 \mathrm{GeV} / c^{2}$. This limit should improve, when more data become available, by refining the background evaluations, by optimizing the analyses in view of such a combination and by combining directly the analyses rather than the limits. All this would require a close collaboration work between the four LEP experiments.

\section{THE HIGGS BOSONS OF THE MSSM}

The basic structure of supersymmetry implies the existence of at least two doublets of Higgs fields, and exactly two doublets in its minimal version. As a consequence, two neutral states ( $h$ and A) may be produced at LEP via the Bremsstrahlung process $\mathrm{e}^{+} \mathrm{e}^{-} \rightarrow \mathrm{hZ}$ on the one hand, and via the associated pair production $\mathrm{e}^{+} \mathrm{e}^{-} \rightarrow \mathrm{hA}$ on the other.

As to the Bremsstrahlung process, the standard searches presented in Section 2 can generally be used without modification, and reinterpreted in the MSSM framework by multiplying the production cross-section by a reduction factor $\sin ^{2}(\beta-\alpha)$. Complementarily, the $\mathrm{e}^{+} \mathrm{e}^{-} \rightarrow \mathrm{hA}$ production has a cross-section proportional to $\cos ^{2}(\beta-\alpha)$, while the subsequent decay branching ratios of $h$ and $A$ are $10 \%$ and $90 \%$ in $\tau^{+} \tau^{-}$and in $\mathrm{b} \overline{\mathrm{b}}$ respectively. Searches for the $\mathrm{hA} \rightarrow \tau^{+} \tau^{-} \mathrm{q} \overline{\mathrm{q}}$ final state had therefore been performed by the four collaborations as early as in 1990 to cover the domain of large $\cos ^{2}(\beta-\alpha)$ values. No candidates were found, allowing the whole kinematically accessible part of this domain to be excluded.

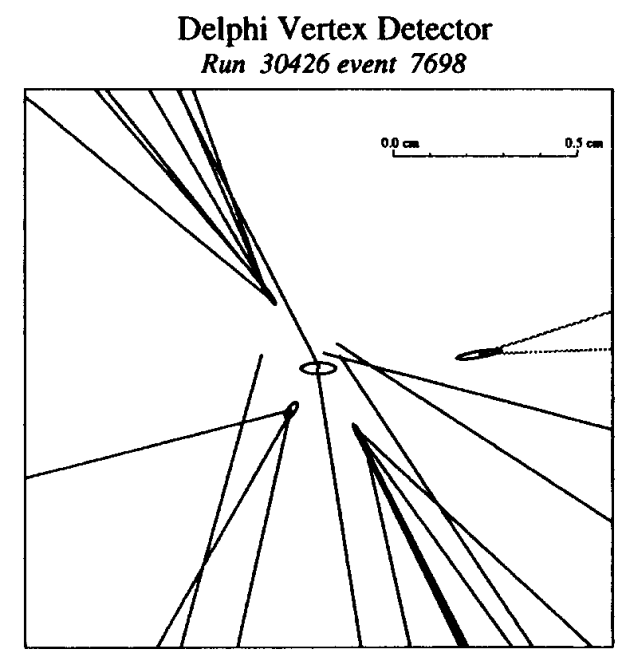

Figure 10. A zoom view of the DELPHI vertex detector for an event with four reconstructed vertices, likely coming from B-meson decays. 

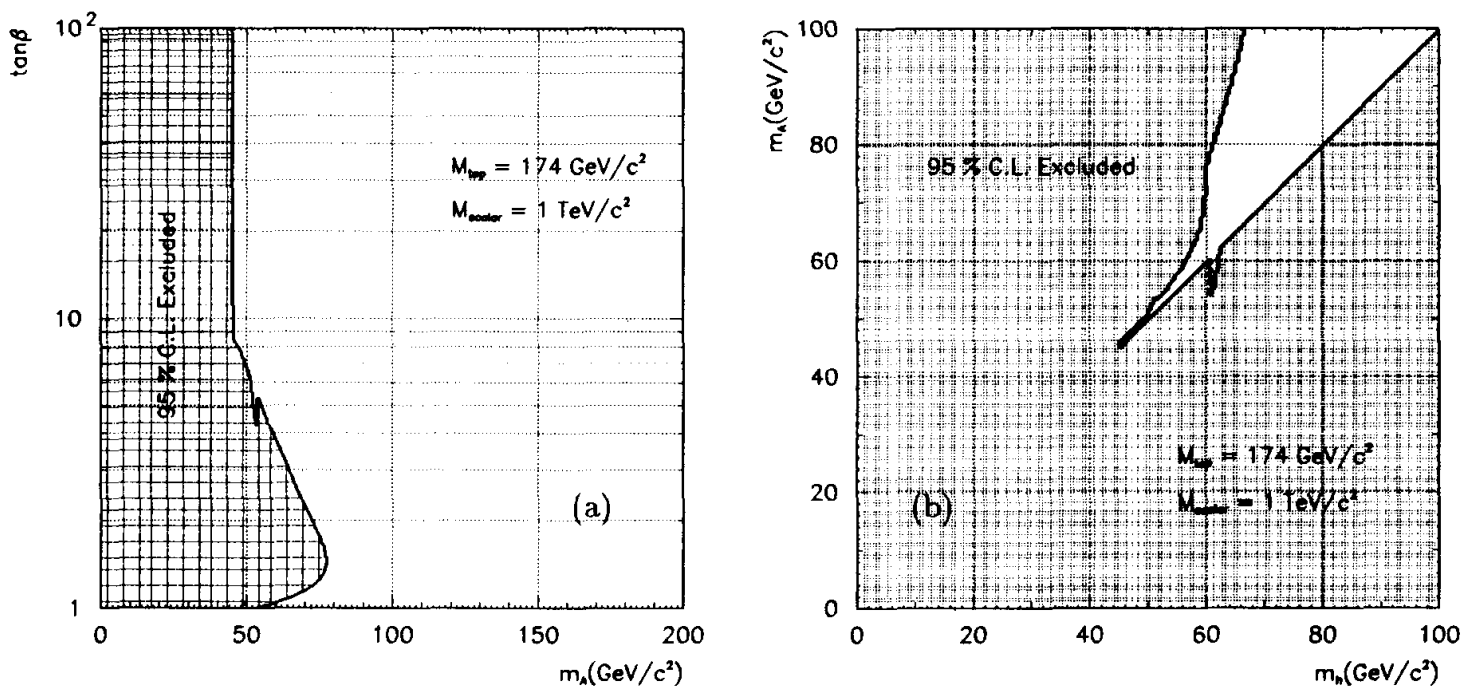

Figure 11. 95\% C.L. excluded domains in the $\left(m_{\mathrm{A}}, \tan \beta\right)$ plane and in the $\left(m_{\mathrm{h}}, m_{\mathrm{A}}\right)$ plane from a combination of the four LEP experiments, determined with a top-quark mass of $174 \mathrm{GeV} / c^{2}$ and a scalar mass of $1 \mathrm{TeV} / c^{2}$.

This has been recently supplemented by a search for the final state $\mathrm{hA} \rightarrow \mathrm{b} \bar{b} b \bar{b}$ developed by DELPHI, using their vertex detector as an efficient and selective b-tagger. A view of an event with four reconstructed vertices in this detector arising from the decay of long-lived particles (such as B-mesons) is presented in Fig. 10. A total of 105 such events were found in the DELPHI data taken until 1992 , to be compared to an expectation of $97 \pm 9 \pm 10$ events from $\mathrm{e}^{+} \mathrm{e}^{-} \rightarrow$ hadrons. This search therefore confirms, in a totally independent way, the negative result of the earlier $\tau^{+} \tau^{-} \mathrm{q} \bar{q}$ searches.

When these searches are combined to the complementary searches for $\mathrm{e}^{+} \mathrm{e}^{-} \rightarrow h Z^{*}$ of the four LEP experiments, limits can be derived either on $m_{\mathrm{h}}$ and $m_{\mathrm{A}}$ or, alternatively, on $m_{\mathrm{A}}$ and $\tan \beta$ (the ratio of the vacuum expectation values of the two Higgs doublets). Using a top-quark mass value of $174 \mathrm{GeV} / c^{2}$ and a universal scalar mass of $1 \mathrm{TeV} / c^{2}$ to compute the important radiative corrections to the Higgs boson masses, the previously developed combination method leads to $m_{\mathrm{h}}>45.5 \mathrm{GeV} / c^{2}$ and $m_{\mathrm{A}}>45 \mathrm{GeV} / c^{2}$, at the $95 \%$ confidence level. The detail of the excluded domains is shown in the $\left(m_{\mathrm{h}}, m_{\mathrm{A}}\right)$ plane (see Fig. 11a) and in the $\left(m_{\mathrm{A}}, \tan \beta\right)$ plane (see Fig. 11b).

\section{THE UNEXPECTED}

The monojet topology, commonly accepted as being background free for new particle searches in $\mathrm{e}^{+} \mathrm{e}^{-}$collisions, has been searched for in particular by the ALEPH collaboration for several years. Such a search has been extensively reported in Ref. [16], and essentially consists in selecting events where one hemisphere is free of any detector activity, with a total momentum transverse to the beam axis in excess of $5 \% \sqrt{s}$.

Three events were selected in the ALEPH data, in good agreement with the expectation of 2.6 events from the so-called conversion processes $\mathrm{e}^{+} \mathrm{e}^{-} \rightarrow \gamma^{*} \nu \tilde{\nu}$, with $\gamma^{*} \rightarrow \overline{f f}$, while no events were retained in any of the Monte Carlo samples simulating $\mathrm{Z}$ decays into a fermion pair or $\gamma \gamma$ processes. 

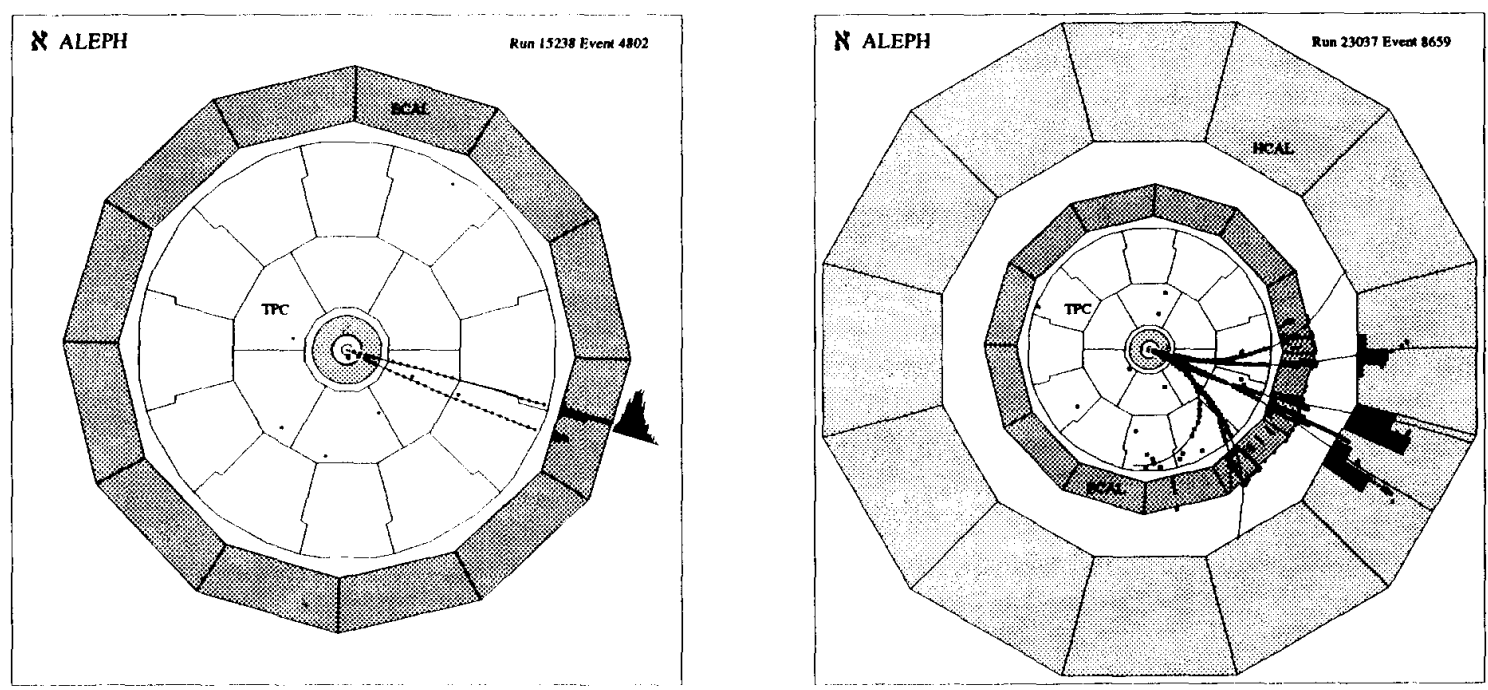

Figure 12. Views of the ALEPH detector for the acoplanar $\mathrm{e}^{+} \mathrm{e}^{-}$pair and for the hadronic monojet.

The visible masses, transverse momenta and invisible recoil masses of these events are given in Table 7. It can be seen that two events, an acoplanar $\mathrm{e}^{+} \mathrm{e}^{-}$pair [17] and a hadronic monojet [16], have unexpectedly large masses and transverse momenta. A view of these two events is shown in Fig. 12.

Table 7

Characteristics of the three monojet-like events selected in the ALEPH data.

\begin{tabular}{rrrr}
\hline Final state & $\begin{array}{r}m_{v i s} \\
\left(\mathrm{GeV} / c^{2}\right)\end{array}$ & $\begin{array}{r}p_{T} \\
(\mathrm{GeV} / \mathrm{c})\end{array}$ & $\begin{array}{r}m_{\text {inv }} \\
\left(\mathrm{GeV} / \mathrm{c}^{2}\right)\end{array}$ \\
\hline $\mathrm{e}^{\mp} \mathrm{e}^{-}$ & 3.3 & 20.3 & 61 \\
Hadronic & 3.2 & 6.6 & 80 \\
Hadronic & 5.3 & 18.5 & 69 \\
\hline
\end{tabular}

Indeed, the probability that such a configuration arise from the conversion process is only $1.0 \%$. When all the diagrams available in the four-fermion generator FERMISV [11] are included, the number of events expected increases as litthe as by 0.06 , but the probability becomes $2.2 \%$. This indicates that further diagrams not taken into account in FERMISV should be considered, namely those involving $W$ exchanges. This has been attempted in Ref. [16], and the result is that a total of 2.75 signal events is expected, with a probability of $4.8 \%$ to show up in an at least as unlikely configuration of masses and transverse momenta.

This rather "small" probability requires to pursue the study of this channel with a more accurate Monte Carlo, with more data accumulated at the $\mathrm{Z}$ peak and, eventually, with a larger centre-ofmass energy.

\section{CONCLUSION}

The most recent updates of the Higgs boson searches at LEP have been presented. In particular, $95 \%$ confidence level limits on the standard model Higgs boson mass ranging from $56.9 \mathrm{GeV} / c^{2}$ to $60.3 \mathrm{GeV} / c^{2}$ have been reported by each of the four LEP experiments, with their data recorded until 1993. A new combination procedure has been used to decide a priori which analysis should or should not be used in the derivation of an unbiased $95 \%$ C.L. combined limit. This combined limit on the standard model Higgs boson mass amounts to $64.3 \mathrm{GeV} / c^{2}$. 
When this result is translated in the framework of the minimal supersymmetric extension of the standard model, limits in the neutral Higgs sector can be obtained: for a top-quark mass of $174 \mathrm{GeV} / c^{2}$ and a universal scalar mass of $1 \mathrm{TeV} / c^{2}, m_{\mathrm{h}}>45.5 \mathrm{GeV} / c^{2}$ and $m_{\mathrm{A}}>$ $45 \mathrm{GeV} / \mathrm{c}^{2}$ at the $95 \%$ confidence level.

On the unexpected side, two monojet events with rather large visible masses and transverse momenta have been reported by ALEPH, with a probability of $\sim 5 \%$ to show up in an at least as unlikely configuration. Although this level of probability does not allow yet any definite conclusion to be drawn, this confirms that any unexpected event, if produced, would immediately be detected at LEP.

\section{ACKNOWLEDGEMENTS}

It is a real pleasure to thank the organizers and the secretariat of the Neutrino ' 94 conference for their hospitality and for having provided us with such an inspiring atmosphere in the lovely surroundings of Eilat. I am grateful to E. Duchovni (OPAL), J.-F. Grivaz (ALEPH), M. Pieri (L3) and V. Ruhlmann (DELPHI) for their cooperation during the preparation of this talk.

\section{REFERENCES}

1. The combination of all LEP precision measurements and of the recent top-mass determination by CDF [14] leads to a standard model Higgs boson mass prediction of $65_{-54}^{+555} \mathrm{GeV} / c^{2}$ at the $95 \%$ confidence level (M. Martinez, private communication).

2. This upper bound is obtained using the recent top-mass determination by CDF [14] and the hypothesis of the tau-bottom mass unification at the GUT scale (M. Carena, private communication).

3. P. Abreu et al., (DELPHI Coll.), "Search for the standard model Higgs boson in $Z$ decays", CERN PPE/94-046, March 1994. The analysis of the 1990 data [18] gives no sensitivity to a Higgs boson mass above $50 \mathrm{GeV} / \mathrm{c}^{2}$ and is therefore not used here.
4. B. Adeva et al., (L3 Coll.), Phys. Lett. 283B (1992), 454.

5. R. Akers et al., (OPAL Coll.), Phys. Lett. 327B (1994), 397.

6. D. Buskulic et al., (ALEPH Coll.), Phys. Lett. 313B (1993), 299.

7. D. Buskulic et al., (ALEPH Coll.), "Improved mass limit for the standard model Higgs boson", ALEPH 94-034, March 1994, Contribution to the Rencontres de Moriond and la Thuile (March 1994), and to the 27th ICHEP, Ref. GLS0568, Glasgow (July 1994).

8. E. Gross, B.A. Kniehl and G. Wolf, "Production and decay of the standard model Higgs boson at LEP II", DESY report 94-035, March 1994.

9. It is to be noted, however, that an update of the $\mathrm{L} 3$ analysis has been presented at the 27 th ICHEP, Ref. GLS0642, Glasgow (July 1994), with a different background study (M. Pieri, private communication).

10. J.-F. Grivaz and F. Le Diberder, "Complementary analyses and acceptance optimization in new particle searches", LAL 92-37, June 1992.

11. J.M. Hilgart, R. Kleiss and F. Le Diberder, Comput. Phys. Commun. 75(1993),191.

12. See for instance D. Treille, "Particle searches", in the Proceedings of the ECHEP, Marseille, France (July 1993), Eds J. Carr and $M$. Perrottet.

13. J.-F. Grivaz and F. Le Diberder, Nucl. Instrum. Methods 333 (1993), 320.

14. F. Abe et al., (CDF Coll.) FERMILAB-PUB 94-116-E, "Evidence for top quark production in $p \bar{p}$ collisions at $\sqrt{s}=1.8 \mathrm{TeV}$, May 1994.

15. G. Borisov et al., (DELPHI Coll.), "Update of the search for the Standard Model Higgs boson", DELPHI 94-85, June 1994, Contribution to the 27th ICHEP, Ref. GLS0304, Glasgow (July 1994).

16. D. Buskulic et al., (ALEPH Coll.), Phys. Lett. 334B (1994), 244.

17. D. Buskulic et al., (ALEPH Coll.), Phys. Lett. 313B (1993), 312.

18. P. Abreu et al., (DELPHI Coll.), Nucl. Phys. B373 (1992), 3. 